\title{
Prototipo de videolaringoscopio: wi-mac-multivision
}

\author{
Moreno Sanchez JE. ${ }^{1}$, De Freitas J. ${ }^{1}$, Silva M. ${ }^{1}$
}

1 Hospital Central de Maracay, Maracay, Venezuela.

Introducción: El manejo de la vía aérea es un reto constante para los anestesiólogos, existen dispositivos que facilitan la intubación orotraqueal entre los cuales se pueden mencionar los videolaringoscopios, en vista de sus altos costos resulta difícil acceder a ellos. El siguiente estudio se trata de un prototipo de videolaringoscopio de bajo costo y alta efectividad que busca solventar un vació en dispositivos para el manejo de la vía aérea en el Hospital Central de Maracay (HCM).

Materiales y Métodos: Diseño no experimental, prospectivo y observacional, bajo la modalidad de proyecto especial y con alcance evaluativo. La población consta de 32 pacientes que ameritaron intubación orotraqueal por parte del servicio de anestesiología y reanimación del HCM con un prototipo de videolaringoscopio a partir de un laringoscopio de Macintosh.

Resultados: El uso del Wi-Mac-Multivision arrojo un 100\% de intubaciones orotraqueales sin presentar ningún tipo de complicación en ellas, solo uno de los pacientes amerito un segundo intento para lograr la intubación orotraqueal. Se logró visualizar las cuerdas vocales según la clasificación de Cormack-Lehane en CL-I 93,7\% y CL-II 6,3\%. En solo 3 casos $(9,4 \%)$ fue necesario el empleo de maniobras externas que mejoraron la visualización de las cuerdas vocales (Maniobras de B.U.R.P.). El tiempo medio necesario para lograr la intubación orotraqueal fue de 29,62 $\pm 7,53$ segundos.

Conclusiones: Wi-Mac-Multivision logra demostrar ser una alternativa a ser empleada por el personal del hospital central para lograr intubaciones orotraqueales, su bajo costo hace fácil replicar dicho dispositivo y beneficiar a más pacientes. Dicho dispositivo tendría ventajas sobre el aprendizaje y capacitación del personal del HCM. Queda por evaluar el número de intubaciones para poder lograr un buen grado de entrenamiento, indicaciones y si puede ser empleado en pacientes vías aéreas difícil, es por ello que queda una línea de investigación abierta en nuestra institución, la cual se espera pueda continuar.

https://doi.org/10.25237/congresoclasa2019.23 